\title{
Laboratory based Retrospective Study to determine the start of SARS-CoV-2 in Patients with Severe Acute Respiratory Illness in Egypt at El-Demerdash tertiary hospitals
}

\section{Sara H.A.Agwa}

Clinical pathology Department, University of Ain Shams, Cairo, Egypt; Faculty of Medicine Ain Shams Research Institute-Molecular Genomic Unit (MASRI-Molecular Genomic Unit)

\section{Hesham Elghazaly}

Oncology Department, Medical Ain Shams Research institute (MASRI), Cairo, Egypt

\section{Sarah El-Nakeep ( $\sim$ Sarahnakeep@gmail.com )}

Department of Internal Medicine, Ain shams University, Cairo, Egypt https://orcid.org/0000-0003-28305052

\section{Ahmad Moustafa}

Department of Biology, American University in Cairo, New Cairo, Egypt

\section{Manal H El-Sayed}

Pediatric Hematology Oncology Department, Ain Shams University, Cairo , Egypt; Faculty of Medicine Ain Shams Research institute- Clinical Research Center (MASRI-CRC)

\section{Hala Hafez}

Clinical pathology Department, University of Ain Shams, Cairo, Egypt; Infection Control unit

\section{Samia abdo}

Clinical pathology Department, University of Ain Shams, Cairo, Egypt; Infection Control unit

\section{Marwa Matboli}

Biochemistry and molecular biology Department, Faculty of medicine Ain Shams university , cairo,

\section{Egypt}

\section{Maha Saad}

Medicinal biochemistry and molecular biology Department, Modern University for Technology and Information

\section{Shaimaa M.Elsayed}

Faculty of Medicine Ain Shams Research Institute-Molecular Genomic Unit (MASRI-Molecular Genomic Unit)

\section{Aya M.Abd Elsamie}

Faculty of Medicine Ain Shams Research Institute-Molecular Genomic Unit (MASRI-Molecular Genomic Unit)

\section{Reham M.Darwish}


Faculty of Medicine Ain Shams Research Institute-Molecular Genomic Unit (MASRI-Molecular Genomic Unit)

\section{Hoda ezz elarab}

Clinical pathology Department, University of Ain Shams, Cairo, Egypt; Infection Control unit

\section{Fatma S. E. Ebeid}

Pediatric Hematology Oncology Department, Ain Shams University, Cairo , Egypt; Faculty of Medicine Ain Shams Research institute- Clinical Research Center (MASRI-CRC)

\section{Sara Makkeyah}

Pediatric Hematology Oncology Department, Ain Shams University, Cairo , Egypt

\section{Mahmoud S El Meteini}

Department of General Surgery, University of Ain Shams

\section{Research Article}

Keywords: SARI, respiratory infections, COVID-19, SARS-CoV-2

Posted Date: September 17th, 2020

DOI: https://doi.org/10.21203/rs.3.rs-76216/v2

License: (c) (1) This work is licensed under a Creative Commons Attribution 4.0 International License. Read Full License 


\section{Abstract}

Purpose: COVID-19 is the most recent pandemic causing morbidity and mortality. Although a part of the pathogens causing SARI, it is unique in causing pulmonary thrombosis and lung fibrosis. Thus different management is needed. We aimed o explore the start of SARS-CoV-2 in preserved SARI samples to know the exact time of its emergence in our hospital, to conduct whole-genome sequencing in positive SARSCoV-2 samples to define its strain. To assess the clinical characteristics of the severe respiratory infection admitted to El-Demerdash hospitals in that same period from our own file reports.

Methods: We conducted a retrospective cohort study among SARI patients who were admitted to Ain Shams university hospitals. preserved nasopharyngeal\& oropharyngeal swabs from 333 SARI patients were used in SARS-CoV-2 detection by RT-Real time PCR. Moreover, whole-genome sequencing of SARSCoV-2 positive samples was performed. Clinical characteristics of the SARI patients were analyzed in the same period to show the relation to morbidity and mortality.

Results: The first case of SARS-CoV-2 was detected in a 6months aged female patient on mid-April 2020, B.1.1.7, clade GR. Co-infection (with both bacterial and viral) was most prevalent in pediatrics than adults, but mortality was higher in adults than pediatrics (18.1\% versus $7.1 \%)$. ICU admission was higher in adults than in the pediatric group (65\% versus $12.8 \%$ ). Co-morbidities were associated with higher mortality and more severe infection. The most common bacterial infection in both adults and pediatrics was Klebsiella pneumoniae, followed by Staphylococcus aureus and Streptococcus pneumoniae.

Conclusion: COVID-19 didn't start till mid-April in the Egyptian hospitals as remarked by this tertiary hospital's data. Co-infection is the most prevalent in children and further research is needed in this area.

\section{Introduction}

SARI is one of the clinical manifestations of COVID-19 disease, and the most lethal. Started in Brazil in the $9^{\text {th }}$ epidemiological week of the outbreak(1). But the exact start of the disease in tertiary hospitals in Egypt is still undetermined as measured by the number of patients needing hospitalization or intensive care admission due this deadly respiratory illness. While SARS-CoV-2 is under the umbrella of SARI, its disease coarse is much different with high risk for thrombosis, lung fibrosis and embolism(2, 3)

As we know different age groups suffer from different pathogenic infections, children and neonates are mainly infected by Streptococcus pneumoniae and RSV in a large cohort done recently (4)while in adults influenza virus is one of the commonest causes of SARI(5). In Japan the prevalence of Streptococcal pneumoniae is $22 \%$ among the healthy children, risk factors include older siblings, older age of the child and day-care attendance(6). The increase prevalence means endemicity of the bacteria and tendency toward bacterial resistance

On the other hand; different microbiological organisms cause different effect on morbidity and mortality. Also concurrent infections could exacerbate the manifestations of the disease(7). 
Here in our study we examined the patients for clinical symptoms and multi-viral and multi-bacterial survey; who had severe respiratory illness in the Pediatrics, Internal Medicine, Geriatrics and Chest Wards and departments'-related ICUs from November 2019 to April 2020.

\section{Materials And Methods}

This is a retrospective cohort study from our hospital records, where we used previously collected stored samples from patients admitted to the specific hospital departments of Ain shams university hospitals (Pediatric hospital, Geriatric hospital, Internal medicine department, Chest department, Pediatrics ICU, Chest ICU and Internal medicine ICU) that are responsible for the management of SARI. The patients are included when they fulfilled the criteria for acute severe respiratory infection, and followed till the end of hospitalization. The clinical outcome is followed either; discharge, transferal or death. We used sequential weekly patches of stored samples to determine the first positive SARS-CoV-2 sample and the patient data of this positive sample.

We used previously collected samples from 333 patients with severe acute respiratory illness; in the period from November 2019 until April 2020, these samples were tested for the presence of SARS-COV-2 by Real-Time PCR followed by Whole viral Genome Sequencing of the positive SARA-COV-2 samples to define the strain .

SARI cases were defined as a respiratory infection with fever of $38^{\circ} \mathrm{C}$, cough, onset within the last 10 days, and hospitalization(8). Records of previously hospitalized patients with SARI provided demographic information, including age, sex, and residence through questionnaires.

\section{Clinical Sample Collection And Processing}

Between November 2020 and April 2020 Oropharyngeal and nasopharyngeal swabs (NP) from hospitalized SARI patients were collected and placed in a centrifuge tube labeled with the patient unique ID and containing $2 \mathrm{~mL}$ viral transport media (VTM). Patients had completed a questionnaire that covered history of fever and/or respiratory symptoms, travelling history, any underlying lung disease, history of chronic or immune-compromised conditions, and final outcome. The records were used retrospectively to assess the patients' clinical characteristics.

The received swabs inside the $15 \mathrm{ml}$ tube were agitated vigorously for 10 seconds using a vortex mixer. VTM were split into 2 pre-labeled, sterile cryovials with the correct patient ID. One cryovial were immediately placed in a freezer $\left(-80^{\circ} \mathrm{C}\right)$, while the other cryovial was used for molecular studies at Faculty of Medicine Ain Shams University Research Institute-

\section{Viral RNA Extraction and SARS-Cov-2 detection by QRT-PCR:}


Laboratory detection of SARS-Cov-2 infection was done in Medical Ain Shams Research Institute ( MASRI ) MOLECULAR GENOMIC LABS Viral RNA isolation was performed using MagMax viral/pathogen nucleic acid isolation kit (ThermoFisher Scientific, USA). Real time reverse transcription polymerase chain reaction (RT-PCR) was used for simultaneous amplification of two target genes, including nucleocapsid protein $(\mathrm{N})$, and open reading frame $1 \mathrm{ab}$ (ORF1ab). COVID119 detection was done using ProLab/CerTest Biotech ViaSure SARS-CoV-2. Real Time PCR detection Kit (VS-NCO296T, CerTest Biotec, S.L, Spain, Catalogue number VS-NCO213L) that was performed upon an Applied Biosystems ${ }^{\mathrm{TM}} 7500$ Fast Real-Time PCR System following the cycling and fluorescence acquisition parameters detailed in manufacturer protocol. Five microliters of RNA that was isolated from clinical samples were used in each real time PCR reaction, with a final volume for $20 \mu \mathrm{L}$. Samples were processed with appropriate; negative, internal and positive controls. Samples were run in duplicate. Real-Time Detection System and analysis was done by Applied biosystem 7500 Real-Time PCR Software v2.0.

Samples were considered negative: $\mathrm{Ct}>38$ or not detected; positive: the amplification curve was sshaped, and the Ct value was $\leq 35$; suspicious: the amplification curve was s-shaped, and $35<\mathrm{Ct} \leq 38$, requiring reexamination. In addition, SARS-CoV-2 nucleic acid test positive interpretation includes, ORF1 $1 \mathrm{ab}$ and $\mathrm{N}$ genes tested positive at the same time in the same specimen; and the ORF1 $\mathrm{ab}$ or $\mathrm{N}$ gene was positive in two different samples of the same patient based on the recommendations of the National Institute for Viral Disease Control and Prevention (China).

\section{Viral Genome sequencing for positive SARS-CoV-2 samples by targeted next-generation sequencing (NGS)}

- RNA preparation ; After viral RNA isolation(previously describes, The RNA concentration was determined by a Qubit ${ }^{\circledR} 2.0$ Fluorometer (Qubit ${ }^{\circledR}$ RNA Assay Kit, Life Technologies) . Reverse Transcription and cDNA synthesis was done after RNA extraction and assessment, RNA was reverse transcribed using the SuperScript ${ }^{T M}$ VILO ${ }^{\text {TM }}$ cDNA Synthesis Kit (Cat. No. 11754050) .

- NGS Library Preparation; Manual library preparation was done using the lon AmpliSeq ${ }^{\text {TM }}$ Library Kit Plus (Cat. Nos. 4488990 )following the user guide, primer pool 1 and 2 target amplification reactions were combined and amplicons Partially digested , barcode adapters were ligated and purified using the lon Xpress ${ }^{\mathrm{TM}}$ Barcode Adapters 1-96 Kit (Cat. No. 447451). Then libraries were quantified using the lon Library TaqMan ${ }^{T M}$ Quantitation Kit (Cat. No. 4468802). Templates were prepared and chips loaded on the ion Chef instrument Using the lon $530^{\text {TM }}$ Kit - Chef (Cat. No. A34461), according to user guide.

- Ion S5 targeted NGS and analysis The libraries were sequenced on the lon GeneStudio S5 Series System platform with an lon AmpliSeq SARS-CoV-2 Research Panel (ThermoFisher Scientific, USA) that contains two pools with amplicons ranging from 125 bp to $275 \mathrm{bp}$ in length and includes $>99 \%$ of the SARS-CoV-2 genome, covering all serotypes. A complete genome (29903 nucleotides) was assembled, with $0.13 \%$ unique mutations to the other viral genomes. Using BLAST against NCBI betacoronavirus database, the closets matches are several sequences bit score of 33479, including 
for example, isolate SARS-CoV-2/human/USA/VA-DCLS-0556/2020 (99.9\%), accession (MT739463). The assembled genome along with the other SAR-CoV-2 genomes obtained and clustered from GISAID were aligned using MAFFT(9).

- Phylogenetic construction: A maximum likelihood phylogenetic tree was constructed using FastTree $(10,11)$ under the general time reversible (GTR) model $(12)$ with gamma distribution (i.e., GTR + ) for the sites rates.

SARS-Cov- 2 nucleic acid tests were done for all the collected swab samples $(n=333)$, but only one case was positive for the test.

This positive case was a female patient 6 months old, her main presenting symptom was fever, and she had hematological malignancy. She is the first Egyptian positive case in Ain Shams University Hospitals that was confirmed on 14 April 2020 and she was negative for all other examined respiratory pathogens. We submitted the genome data on the GISAID website accession number:

\section{>hCoV-19/Egypt/MASRI-030/2020|EPI_ISL_482775|2020-04-14}

- Reports of results of the routine nasopharyngeal swab and throat swab that were taken from all the patients with suspected SARI for culture analysis were included for statistical analysis.

- Reports of previous detection of viral infection was through lab and clinical data, where the patients were assessed for low neutrophil count and high lymphocytic count, exaggeration of fever or cough after bacterial infection is diagnosed or associated enlarged lymph nodes.

\section{Ethical standards}

Prior to study initiation, the study protocol was reviewed and approved by the Ethical Committee of AinShams University. Reports from hospital records were used. Samples used in this study were previously ethically approved with informed patients' consent in an ongoing project: (We have gathered nasopharyngeal\& oropharyngeal swabs from 333 SARI patients which was used for molecular detection of 33 respiratory pathogenic (viral \& bacterial) targets). Specific national laws have been observed

\section{Statistical methodology:}

The data was analyzed using SPSS version 23.0 from IBM (IBM Corporation, Armonk, NY, USA) and Python Programming language 3.8.3. Graphs were drawn using Microsoft Word.

For numerical values median and interquartile range were calculated. For categorical values number and percentage are presented. $P$ value was considered significant if $<0.05$. Fisher's Exact test was done for 
cross tabulation of binary data in between two groups, while Chi square test was done for binary data with more that two groups, and Chi Square for trend test for ordered categorical data.

Multivariate analysis was done using binary logistic regression to formulate a predictive model for the clinical outcome.

\section{Results}

This cohort study was conducted in Ain Shams University Hospitals on 333 patients, 273 pediatrics and 60 adults. We explored the presence of SARS-COV- 2 in all these samples, also we tested the clinical data and the effect of bacterial versus viral or coinfection on the clinical outcome.

The first COVID SARS-CoV-2 case detected was B.1.1.7, clade GR. This sample was shown with bigger font in the GH clade, which seems to be a more infectious clade than the others. Figure showing the phylogenic tree of this sample across the data currently present in GISAID is shown in figure 5 . The sample was from the pediatric ward on 18 April 2020 of a female patient 6 months of age.

A complete genome (29903 nucleotides) was assembled, with $0.13 \%$ unique mutations to the other viral genomes. Using BLAST against NCBI betacoronavirus database, the closets matches are several sequences bit score of 33479, including for example, isolate SARS-CoV-2/human/USA/VA-DCLS0556/2020 (99.9\%), accession (MT739463). The phylogenetic analysis placed our assembled genome within GISAID Clade GH (Figure 5).

Figure 2 showed the history of chronic clinical illnesses associated with the SARI (showed as percentage not absolute numbers)

There was a statistical significant difference between the two groups regarding the mono versus coinfection as shown in figure 3 .

Demographic data was analyzed for prevalence and percentage. The mean age in children was divided in two categories below or equal 2 years and above two years, as most of our pediatric sample was in those age groups. Also, as for other relevant clinical data as influenza vaccination and antiviral intake are presented in table 1.

We assessed the relation between viral infection, bacterial infection and co-infection and the various clinical comorbidities and clinical presentation and outcome in table 2.

The distribution of the study population according to their governorate place of inhabitance is described in figure 1, as this is a tertiary hospital we find patients from all across the country. But as we notice that due to the geographical area most patients are from Cairo.

Regarding the clinical data and comorbidities we found that the pediatrics group had more prevalence of associated Asthma and chronic hematological disease. While adults has more prevalence of diabetes, 
chronic respiratory disease, chronic renal illness, immunodeficiency, chronic cardiac disease and chronic liver disease figure 2.

ICU admission and ICU transferal from ward were markedly higher in the adult group than the pediatric group as shown in table 1. Most common detected organism in swab and throat cultures was Klebsiella pneumoniae as shown in table 1.

Although the temperature $>38$ at presentation was not correlated with the number of total infections, the unique distribution was apparent in figure 4.

A multivariate model by logistic regression to predict the primary outcome death or discharge from the hospital was presented in table 3.

\section{Discussion}

Our study is a retrospective cohort study assessing the cause for SARI from November 2019 till April 2020. We found that the first detected SARS-CoV-2 sample was on 14 April 2020 of a female pediatric patient 6 months of age with hematological malignancy. She presented with high-grade fever $>40^{\circ} \mathrm{C}$ and severe cough. She didn't need ICU admission or ventilation and was isolated in pediatric ward and received medical treatment, and discharged later after improvement and negative swab.

In accordance with our study about the start of dispersion of infection of SARS-CoV-2, another Scottish retrospective study examining upper respiratory samples stored from December to February found that the first positive sample for SARS-CoV-2 was on the first of March(13). Similar retrospective studies for exploring SARS-CoV-2 among different populations were conducted with similar results. Deslandes group (14) tested 14 nasopharyngeal samples taken between December 2019 and January 2020. They detected one positive case from late December. Also, Hogen et al., (15) retrospectively tested 2,888 bronchoalveolar lavage and nasopharyngeal samples in the period between January and February in San Francisco. Two cases were positive from late February, which coincide with the first reported cases in the nearby area in in France. However, Another research group (16)demonstrated no positives in 1,700 samples retrospectively tested from November to December 2019 in the San Francisco Bay Area. Rachael et al., found no SARS-CoV-2 positivity in the 174 respiratory samples collected from Greater Glasgow and Clyde ICUs between December 2019 and February 2020 before the appearance of first reported Scottish case(13).

Cancer patients show higher risk and poor outcomes from the SARS-CoV-2 infection(17). Of note, the first reported Egyptian case in Ain Shams university hospital was from pediatric oncology department due to hematological malignancy. A recent study of 18 patients with previous cancer(18), in a nationwide cohort of COVID-19 cases, declared that patients with cancer had a higher risk of severe clinical features with case fatality rate up to $5.6 \%$ among cancer patients in comparable with $2.3 \%$ among the general population(19). 
We found in our study that the comorbidities were different in the adults and the pediatric group; were chronic cardiac illness and COPD along with diabetes and renal disease were more prevalent in the adult group. On the other hand chronic hematological illness and Asthma were more prevalent in the pediatric group. Comparative to a previous SARI studies were they found that chronic cardiac disease and respiratory illness were the most common association with SARI but they didn't specify the age groups(20). Also a study conducted in Chilie found that adults are more prone to SARI when they suffered from cardiac and respiratory illnesses(5). An Egyptian study survey was conducted recently in Assuit and included pediatric and adult groups from 2010-2014 by Hatem et al.,they found that 53\% of patients had co-morbidities mostly in adults not children, and in the form of chronic lung diseases $43 \%(21)$.

This indicates that co-morbidities in adults is more common than children owning to the risk factors as aging, smoking, long term illnesses, while in children most of the comorbidities is related to hereditary, nutritional or environmental risk factors $(22,23)$.

While vaccination is slightly more prevalent in the adult group (3.1\%) than the pediatric group (1.4\%), there is no real difference, this could be due to low influenza vaccination rates in the high risk and vulnerable groups in Egypt; although there is ongoing plan to extend the care to overcome the limitations encountered(24).

In our study the prevalence of Streptococcal pneumoniae in nasopharyngeal and throat swabs were $43.2 \%$ in pediatrics and $10 \%$ in adults, thus more prevalent in the pediatric group. Streptococcal pneumoniae a known pathogen to cause meningitis, along with pharyngitis, appears to be common in children. Although vaccination and proper antibiotic regimens appear to be effective, the emergence of resistant strains are affecting the overall course of the infection(25). Increasing the number of siblings in the household is an important risk factor, also increase in the density of bacterial load in the nasopharyns, where both seems to be affected by the hygiene of the patient and the socioeconomic class(26). On the other hand; a recent interesting study noticed the effect of respiratory syncytial virus on increasing the rate of bacterial streptococcal co-infection in children(27), this may be attributed to the enhanced attachment to the epithelium and facilitation of immune invasion in viral infection(28).

Unfortunately; the bacterial infection in both pediatric and adults groups with Klebsiella pneumoniae was common with 129 (47.3\%) cases and 22 (36.7\%) cases respectively. In addition to being a cause for severe respiratory illness mandating hospitalization, this strain is more prone to multidrug resistance and complications especially in ICU patients(29).

The prediction model we used explains the effect of diabetes, which is a cause for relative immunedeficiency in adults and its association with increased infection rate in general as a predictor for increased mortality in SARI patients (30). And since most of the diabetic patients in our study are present in the adult group that explains the higher mortality in adult group $(18.1 \%)$ relative to the pediatric group (7.1\%) with odds ratio $\mathrm{OR}=5.7$. 
In addition the need for ventilation as an indication for severity of the respiratory illness was a strong predictor for mortality with $\mathrm{OR}=316$. Number of bacterial infection increased the odds of mortality as we explained the role of co-infection in affecting the immunity response to infection.

Our first detected sample was B.1.1.7, clade GR. There are three distinguished variants according to amino acid mutations are detected in SARS-CoV-2 genome; these are A, B and C (31).

On the other hand, a whole genome study of the viral clades of patients imported to China revealed the prevalence of $\mathrm{G}$ clade among them, the variant present in the $\mathrm{G}$ clade is D614G in the S-protein which is more predominant in Europe and characterized by increased severity of the disease (32). As the aspartic acid (D) amino acid appears to be substituted by glycine (G) amino acid; changing the highly glycosylated region of the spike protein thus affecting its pathogenicity as it is the region responsible for viral entry and identification (33). This might explain the difference in severity of infection in different clades and different geographical regions.

\section{References}

1. Bastos LS, Niquini RP, Lana RM, Villela DAM, Cruz OG, Coelho FC, et al. COVID-19 and hospitalizations for SARI in Brazil: a comparison up to the 12th epidemiological week of 2020. Cadernos de saude publica. 2020;36(4):e00070120.

2. Bompard F, Monnier H, Saab I, Tordjman M, Abdoul H, Fournier L, et al. Pulmonary embolism in patients with Covid-19 pneumonia. The European respiratory journal. 2020.

3. George PM, Wells AU, Jenkins RG. Pulmonary fibrosis and COVID-19: the potential role for antifibrotic therapy. The Lancet Respiratory medicine. 2020.

4. Pneumonia Etiology Research for Child Health Study G. Causes of severe pneumonia requiring hospital admission in children without HIV infection from Africa and Asia: the PERCH multi-country case-control study. Lancet. 2019;394(10200):757-79.

5. Fica A, Sotomayor V, Fasce R, Dabanch J, Soto A, Charpentier P, et al. Severe acute respiratory infections (SARI) from influenza in adult patients in Chile: the experience of a sentinel hospital. Revista panamericana de salud publica = Pan American journal of public health. 2019;43:e1.

6. Ueno M, Ishii Y, Tateda K, Anahara Y, Ebata A, lida M, et al. Prevalence and risk factors of nasopharyngeal carriage of Streptococcus pneumoniae in healthy children in Japan. Japanese journal of infectious diseases. 2013;66(1):22-5.

7. Griffiths EC, Pedersen AB, Fenton A, Petchey OL. The nature and consequences of coinfection in humans. J Infect. 2011;63(3):200-6.

8. WHO. Global epidemiological surveillance standards for influenza [Internet]. WHO surveillance case definitions for ILI and SARI. Geneva, Switzerland: World Health Organization. 2014;[cited 29 June 2020]. 
9. Katoh K, Misawa K, Kuma K-i, Miyata T. MAFFT: a novel method for rapid multiple sequence alignment based on fast Fourier transform. Nucleic Acids Res. 2002;30(14):3059-66.

10. Price MN, Dehal PS, Arkin AP. FastTree: Computing Large Minimum Evolution Trees with Profiles instead of a Distance Matrix. Molecular Biology and Evolution. 2009;26(7):1641-50.

11. Price MN, Dehal PS, Arkin AP. FastTree 2--approximately maximum-likelihood trees for large alignments. PloS one. 2010;5(3):e9490-e.

12. Masatoshi et al. Molecular Evolution and Phylogenetics. . USA: Oxford University Press; 2000.

13. Tomb RM, MacLean DAR, Gunson PRN. Retrospective screening for SARS-CoV-2 in Greater Glasgow and Clyde ICUs between December 2019 and February 2020. J Infect. 2020:S0163-4453(20)30403-5.

14. Deslandes A, Berti V, Tandjaoui-Lambotte Y, Alloui C, Carbonnelle E, Zahar JR, et al. SARS-CoV-2 was already spreading in France in late December 2019. International Journal of Antimicrobial Agents. 2020;55(6):106006.

15. Hogan CA, Sahoo MK, Pinsky BA. Sample Pooling as a Strategy to Detect Community Transmission of SARS-CoV-2. JAMA. 2020;323(19):1967-9.

16. Hogan CA, Garamani N, Sahoo MK, Huang C, Zehnder J, Pinsky B. Retrospective Pooled Screening for SARS-CoV-2 RNA in late 2019. medRxiv. 2020:2020.05.14.20102079.

17. Zhang L, Zhu F, Xie L, Wang C, Wang J, Chen R, et al. Clinical characteristics of COVID-19-infected cancer patients: a retrospective case study in three hospitals within Wuhan, China. Annals of Oncology. 2020;31(7):894-901.

18. Liang W, Guan W, Chen R, Wang W, Li J, Xu K, et al. Cancer patients in SARS-CoV-2 infection: a nationwide analysis in China. Lancet Oncol. 2020;21(3):335-7.

19. Wu Z, McGoogan JM. Characteristics of and Important Lessons From the Coronavirus Disease 2019 (COVID-19) Outbreak in China: Summary of a Report of 72314 Cases From the Chinese Center for Disease Control and Prevention. JAMA. 2020;323(13):1239-42.

20. Chakhunashvili G, Wagner AL, Power LE, Janusz CB, Machablishvili A, Karseladze I, et al. Severe Acute Respiratory Infection (SARI) sentinel surveillance in the country of Georgia, 2015-2017. PloS one. 2018;13(7):e0201497.

21. Hatem A, Mohamed S, Abu Elhassan UE, Ismael EAM, Rizk MS, El-Kholy A, et al. Clinical characteristics and outcomes of patients with severe acute respiratory infections (SARI): results from the Egyptian surveillance study 2010-2014. Multidisciplinary respiratory medicine. 2019;14:11.

22. Kinyoki DK, Manda SO, Moloney GM, Odundo EO, Berkley JA, Noor AM, et al. Modelling the Ecological Comorbidity of Acute Respiratory Infection, Diarrhoea and Stunting among Children Under the Age of 5 Years in Somalia. Int Stat Rev. 2017;85(1):164-76.

23. Rivero-Calle I, Cebey-López M, Pardo-Seco J, Yuste J, Redondo E, Vargas DA, et al. Lifestyle and comorbid conditions as risk factors for community-acquired pneumonia in outpatient adults (NEUMO-ES-RISK project). BMJ Open Respir Res. 2019;6(1):e000359-e. 
24. Abubakar A, Melhem N, Malik M, Dbaibo G, Khan WM, Zaraket H. Seasonal influenza vaccination policies in the Eastern Mediterranean Region: Current status and the way forward. Vaccine. 2019;37(12):1601-7.

25. Koliou MG, Andreou K, Lamnisos D, Lavranos G, lakovides P, Economou C, et al. Risk factors for carriage of Streptococcus pneumoniae in children. BMC pediatrics. 2018;18(1):144.

26. Fadlyana E, Dunne EM, Rusmil K, Tarigan R, Sudigdoadi S, Murad C, et al. Risk factors associated with nasopharyngeal carriage and density of Streptococcus pneumoniae, Haemophilus influenzae, Moraxella catarrhalis, and Staphylococcus aureus in young children living in Indonesia. Pneumonia. 2018;10:14.

27. Brealey JC, Chappell KJ, Galbraith S, Fantino E, Gaydon J, Tozer S, et al. Streptococcus pneumoniae colonization of the nasopharynx is associated with increased severity during respiratory syncytial virus infection in young children. Respirology. 2018;23(2):220-7.

28. Wang JH, Kwon HJ, Jang YJ. Rhinovirus enhances various bacterial adhesions to nasal epithelial cells simultaneously. The Laryngoscope. 2009;119(7):1406-11.

29. Paczosa MK, Mecsas J. Klebsiella pneumoniae: Going on the Offense with a Strong Defense. Microbiology and molecular biology reviews : MMBR. 2016;80(3):629-61.

30. Li S, Wang J, Zhang B, Li X, Liu Y. Diabetes Mellitus and Cause-Specific Mortality: A PopulationBased Study. Diabetes \& metabolism journal. 2019;43(3):319-41.

31. Forster P, Forster L, Renfrew C, Forster M. Phylogenetic network analysis of SARS-CoV-2 genomes. Proceedings of the National Academy of Sciences of the United States of America. 2020;117(17):9241-3.

32. Jia Y, Yang C, Zhang M, Yang X, Li J, Liu J, et al. Characterization of eight novel full-length genomes of SARS-CoV-2 among imported COVID-19 cases from abroad in Yunnan, China. J Infect. 2020;81(2):e96-e8.

33. Brufsky A. Distinct viral clades of SARS-CoV-2: Implications for modeling of viral spread. Journal of medical virology. 2020.

\section{Tables}

\section{Table 1 showing the demographic base line data in the two groups:}




\begin{tabular}{|c|c|c|}
\hline Clinical data & Pediatrics $\mathbf{N}=273$ & $\begin{array}{l}\text { Adults } \\
\text { (3groups) } \quad \mathrm{N}= \\
60\end{array}$ \\
\hline Age (median and IQR) & $\begin{array}{l}<\text { or }=\text { 2years: } \\
6 \text { months }(2-13) \\
>2 \text { years: 5years } \\
(3-7)\end{array}$ & $\begin{array}{l}63 \text { years(55- } \\
76.5)\end{array}$ \\
\hline Sex & Male: $122(56.7 \%)$ & Male: $18(50 \%)$ \\
\hline & $\begin{array}{l}\text { Female: } \\
(43.3 \%) \\
\text { Missing } 58\end{array}$ & $\begin{array}{l}\text { Female: } \quad 18 \\
(50 \%) \\
\text { Missing } 24\end{array}$ \\
\hline ICU admission & $35(12.8 \%)$ & $\begin{array}{l}\text { Total: } 39(65 \%) \\
\text { Medicine: } 11 \\
\text { Geriatric: } 13 \\
\text { Chest: } 15\end{array}$ \\
\hline Ward admission & $238(87.2 \%)$ & $\begin{array}{l}\text { Total: } 21(35 \%) \\
\text { Medicine: } 3 \\
\text { Geriatric: } 14 \\
\text { Chest: } 4\end{array}$ \\
\hline Outcome: & Total: 210 & Total: 33 \\
\hline Death & $15(7.1 \%)$ & $6(18.1 \%)$ \\
\hline Discharge & $192(91.4 \%)$ & $27(81.8 \%)$ \\
\hline Transfer & $3(1.4 \%)$ & 0 \\
\hline \multicolumn{3}{|c|}{ The organisms in swab and throat cultures } \\
\hline $\begin{array}{l}\text { Klebsiella Pneumoniae infection } \\
\text { detected in blood culture }\end{array}$ & $129 / 273$ & $22 / 60$ \\
\hline Streptococcus pneumoniae & $118 / 273$ & $6 / 60$ \\
\hline Staphylococcus aureus & $91 / 273$ & $11 / 60$ \\
\hline Needed ventilation & $20 / 194(10.3 \%)$ & $7 / 21(33.3 \%)$ \\
\hline Fever $>38 \mathrm{C}$ & $\begin{array}{l}\text { Yes: } 185(85.6 \%) \\
\text { No: } 31(14.4 \%)\end{array}$ & $\begin{array}{l}\text { Yes: } 17(53.1 \%) \\
\text { No: } 15(46.9 \%)\end{array}$ \\
\hline $\begin{array}{l}\text { Temperature at admission: } \\
<\text { or }=37.5 \\
37.5-39 \\
>39\end{array}$ & $\begin{array}{l}82(34.2 \%) \\
96(40 \%)\end{array}$ & $\begin{array}{l}22(61.1 \%) \\
13(36.1 \%)\end{array}$ \\
\hline
\end{tabular}




\begin{tabular}{|l|l|l|} 
& & \\
& & $1(2.7 \%)$ \\
& & \\
\hline Cough & Yes: $187(86.6 \%)$ & Yes: $33(100 \%)$ \\
& No: 29 & No: 0 \\
\hline Transfer to ICU & Yes: $29(13.4 \%)$ & Yes: $19(61.3 \%)$ \\
& No: 187 & No: 12 \\
\hline Influenza vaccine & Yes: $3(1.4 \%)$ & Yes: $1(3.1 \%)$ \\
& No: 205 & No: 31 \\
\hline
\end{tabular}

Table 2 showing the Difference between the two groups regarding having viral-bacterial and co-infection associated with each of the following variables

\begin{tabular}{|l|l|l|}
\hline Co-morbidities & Significance & P-value \\
\hline Chronic cardiac & Significant & 0.006 \\
\hline Asthma & Non-Significant & 0.576 \\
\hline Chronic respiratory disease & Significant & 0.003 \\
\hline Chronic liver disease & Non-Significant & 0.086 \\
\hline Diabetes & Significant & 0.008 \\
\hline Chronic neurological & Non-Significant & 0.346 \\
\hline Chronic renal & Non-Significant & 0.9 \\
\hline Chronic hematological & Non-Significant & 0.546 \\
\hline Immunodeficiency & Significant & 0.035 \\
\hline Clinical data: & \multicolumn{2}{|l|}{} \\
\hline Fever >38C & Significant & 0.039 \\
\hline Cough & Significant & 0.012 \\
\hline Death/discharge & Non-Significant & 0.411 \\
\hline Admission to ICU & Non-Significant & 0.152 \\
\hline Transfer to ICU & Significant & 0.000019 \\
\hline Pediatrics/adults & Significant & $5 \times 10-17$ \\
\hline
\end{tabular}

Table 3 showing logistic regression with multivariate analysis predictive of the death/discharge outcome: 


\begin{tabular}{|c|c|c|}
\hline Variables & Significance & OR \\
\hline 1- Patient Ventilated & 0.000 & 316.350 \\
\hline 2- Diabetes & 0.267 & 5.768 \\
\hline 3- Number of bacterial infections & 0.088 & 1.889 \\
\hline Constant & $<0.001$ & 0.002 \\
\hline
\end{tabular}

\section{Declarations}

Acknowledgements: Mohamed El-Nakeep MSc., Ain Shams University conducted the statistical methodology and analysis.

Funding: all the authors declare that they didn't receive funding for the research.

Ethics approval: the study protocol was approved by AinShams university ethical committee.

Consent to participate: informed written consent was taken from the patients upon admission to the hospitals.

Consent for publication:N/A

Conflicts of interest/Competing interests: all the authors declare that they have no conflict of interest

Availability of data and material: data available in the form of excel sheet and word format, FASTA format for the COVID-19 case is available.

Code availability: N/A

\section{Figures}




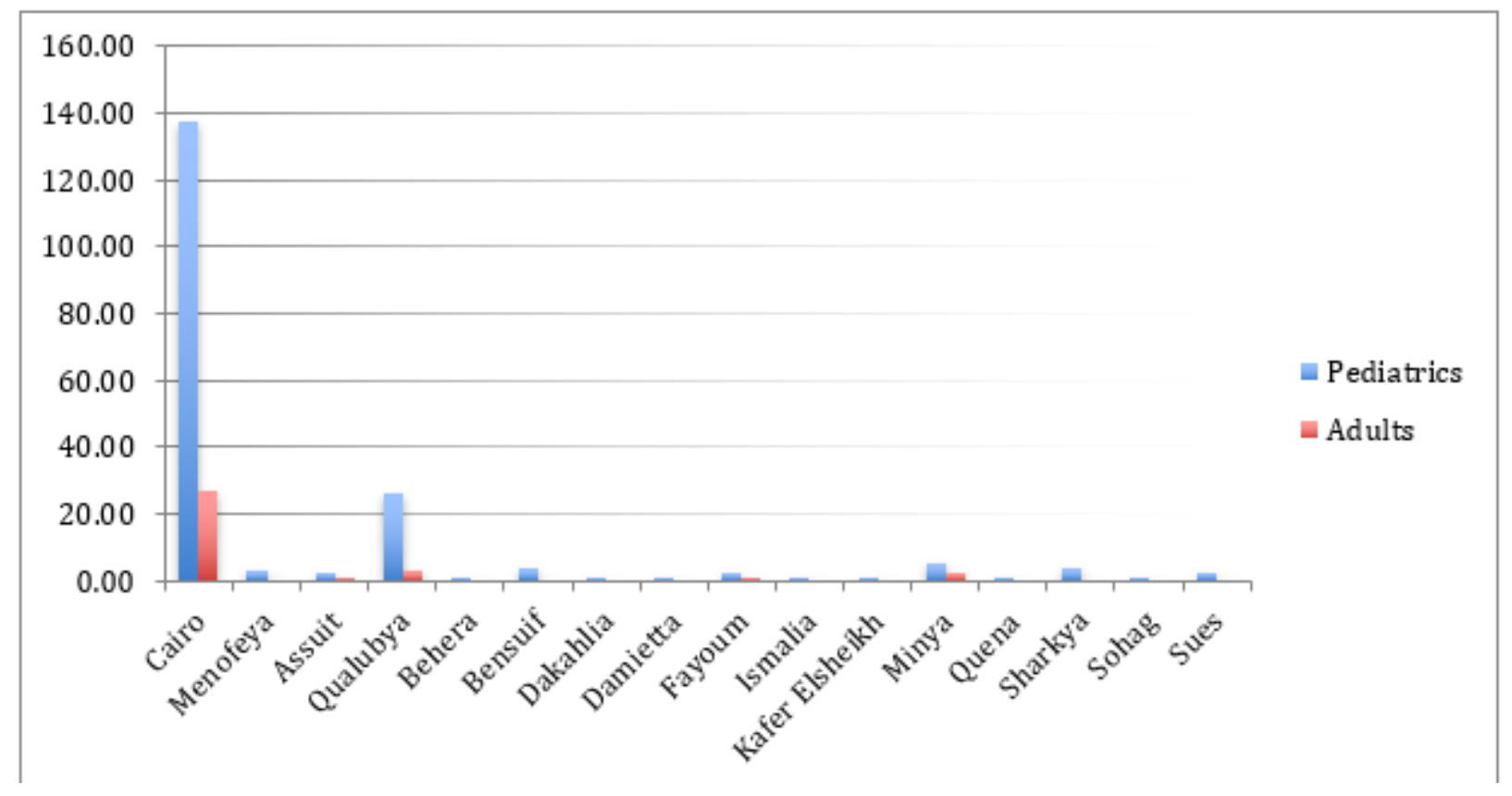

Figure 1

showing the distribution across governorate

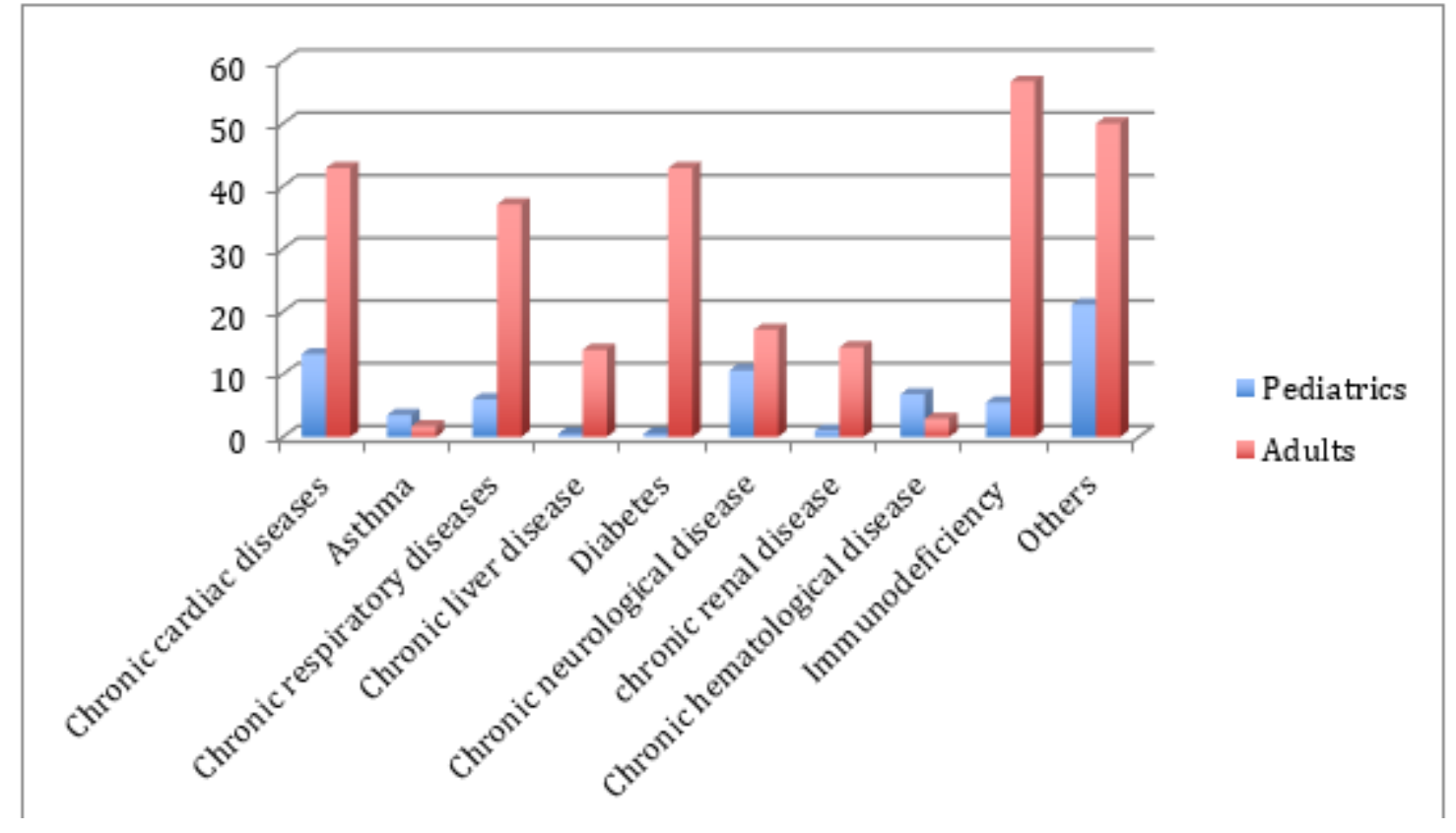

Figure 2

showing prevalent percentage of the history of different chronic illness in the two groups (the bars showing percentage in each group not absolute numbers) 


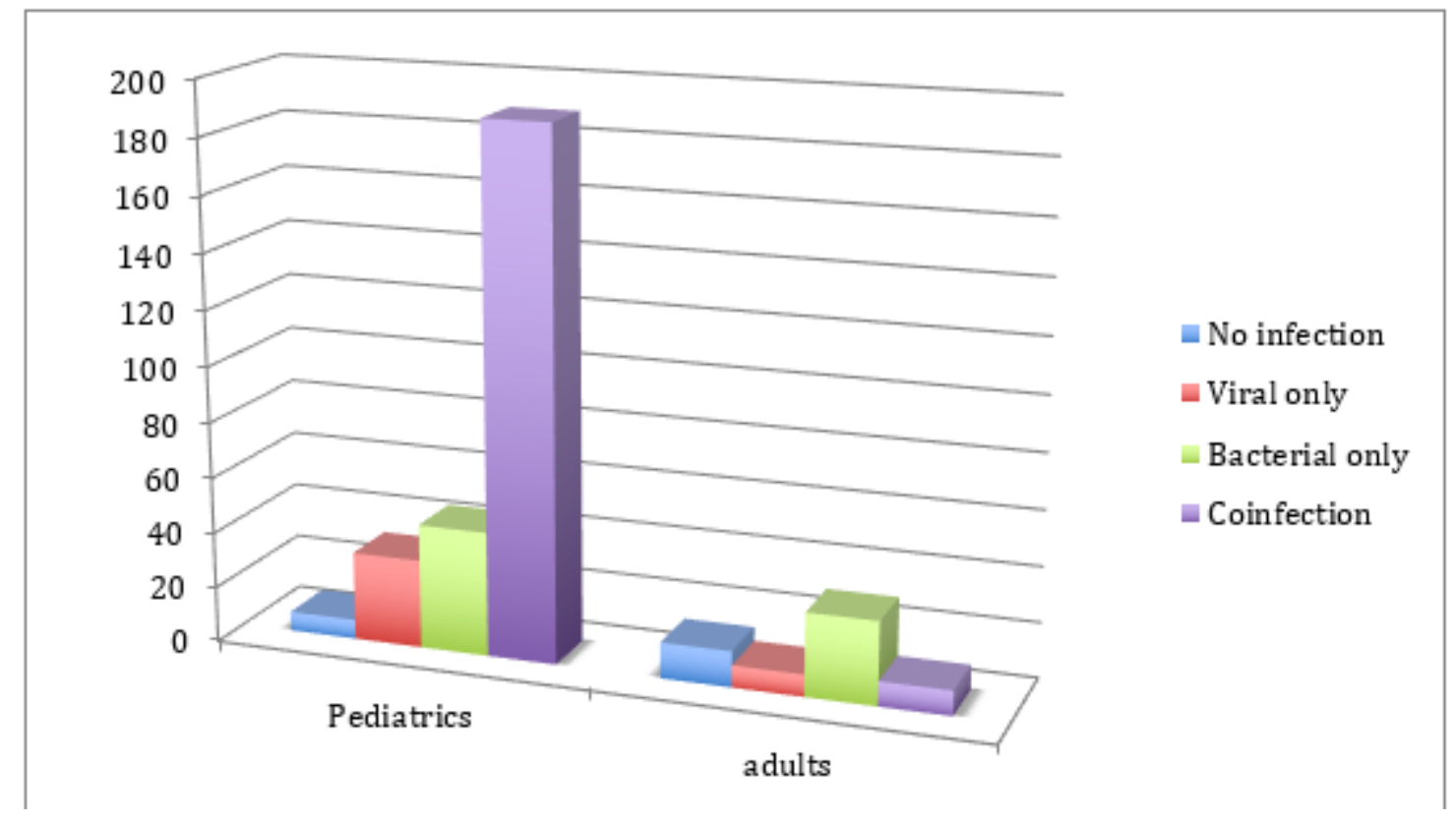

Figure 3

clustered bar chart showing distribution of no infection, the Bacterial only, viral only or co-infection:

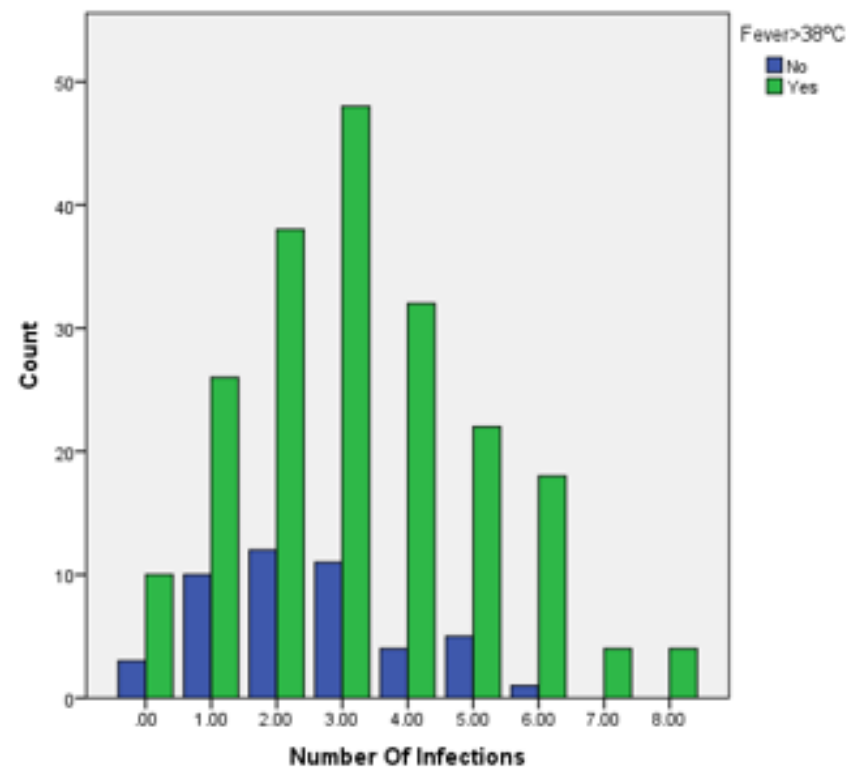

\section{Figure 4}

showing Distribution of fever $>38 \mathrm{C}$ in relation to number of total infections 


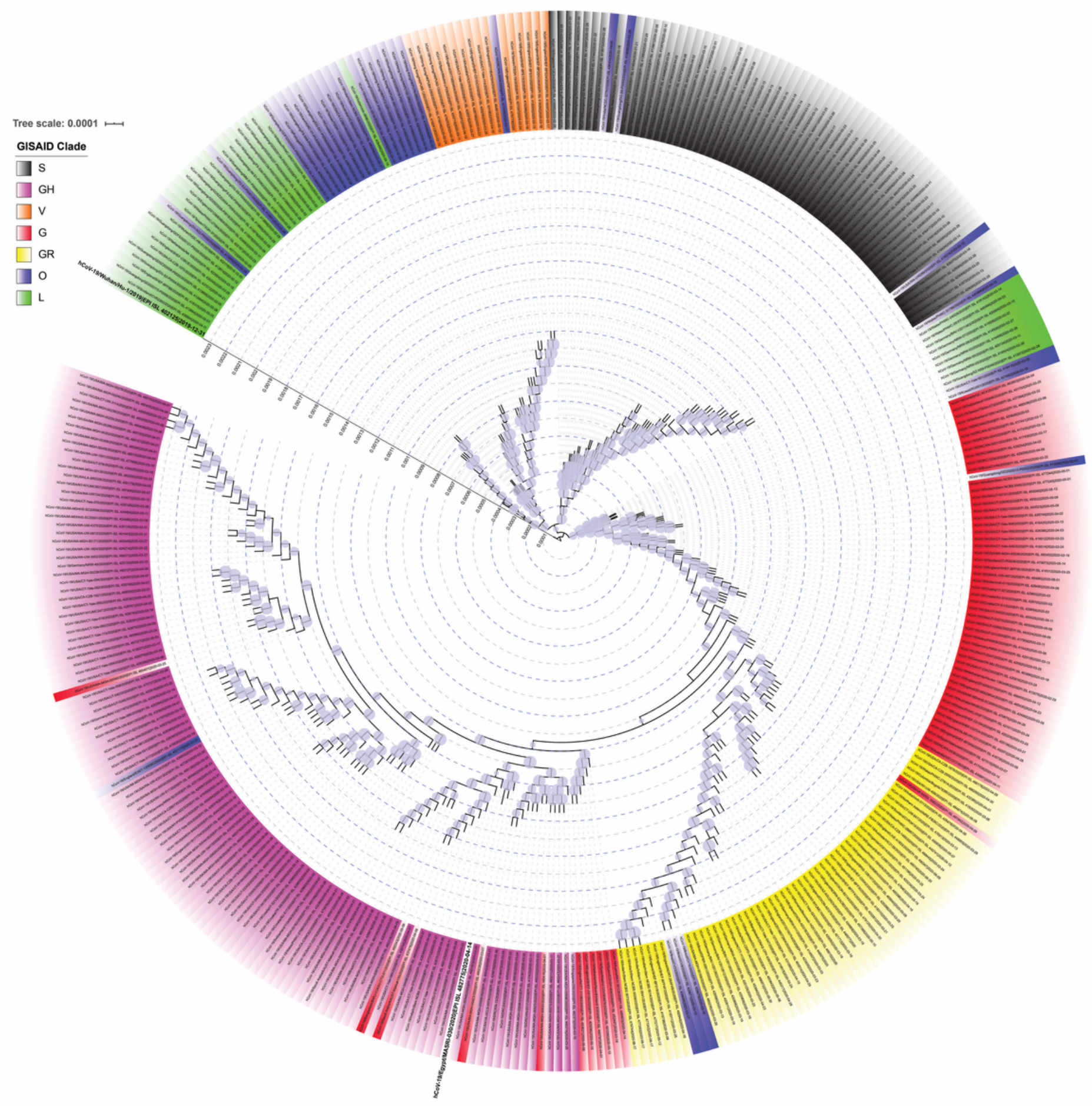

Figure 5

showing a phylogenetic tree of the single sample detected in April 2020 in comparison to the whole GISAID data. 